\title{
ANALYSIS OF THE SELF-ORGANIZING MAP-BASED INVESTMENT STRATEGY
}

\author{
Piotr Kossakowski ${ }^{1)}$, Piotr Bilski ${ }^{2)}$ \\ ${ }^{1)}$ Warsaw University of Life Sciences, ul. Nowoursynowska 159, 02-776 Warsaw, Poland, \\ kossakowski.piotr@gmail.com \\ ${ }^{2)}$ Institute of Radioelectronics and Multimedia Technology, ul. Nowowiejska 15/19, 00-665 Warsaw, Poland, \\ pbilski@ire.pw.edu.pl
}

\begin{abstract}
The following paper presents the application of Self-Organizing Maps (SOM) to construct and apply investment strategy on the stock market. Characteristics of this type of neural network and their influence on the investment strategy performance are verified. Considered parameters include the SOM size, here connected to the size of the training set (number of examples). The average number of patterns per neuron was selected as the appropriate measure. Other aspects of the SOM analysis included conscience mechanism, which allows more neurons to be stimulated during the learning process, method of weights updating, determining the number of stimulated neurons. Additionally, the impact of the correlation between features was verified to eliminate redundant ones. Performance of each designed network was verified against the simple investment strategy, generating "buy", "sell" and "hold" signals based on the average Rate of Return (RoR). Results show that SOMs with the conscience mechanism outperform their simpler configurations. Elimination of correlated data also improves performance of the SOM-based investment strategy. Copyright (C) Research Institute for Intelligent Computer Systems, 2017. All rights reserved.
\end{abstract}

Keywords: stock market, investment strategy, Self-Organizing Maps, prediction, unsupervised learning.

\section{INTRODUCTION}

Customers of the stock markets cannot rely on their intuition or blind luck when making investment decisions. Their decisions have to be as certain as possible to maximize profits and minimize losses. Effective investments can be supported in many ways. The most rational ones include the technical and fundamental analysis or the usage of computer applications and strategies specifically created for this purpose. Application of Artificial Intelligence (AI) methods for automatic forecasting, classification and processing of data is currently a standard approach. One of the popular learning and decision making algorithms are neural networks.

In this paper the unsupervised learning networks, i.e. Self-Organizing Maps (SOM) are used to find dependencies within time series constructed by the prices of shares in the subsequent days. The networks' characteristics affect performance of the investment strategy built on data classified by them. Parameters of SOM are verified to find the optimal configuration in the particular case. The paper contains extended set of experiments using the designed network, initially presented in [1].

The paper is organized as follows. The considered problem is described in Section 2, while the methodology of the conducted experiments is in Section 3. The investment strategy used for generating "buy" and "sell" signals is introduced in Section 4. Detailed information about experimental setup is provided in Section 5. Results of investigations are presented in Section 6 . Conclusions and possible future applications of the proposed methodology are in Section $7^{1}$.

\section{PROBLEM DEFINITION}

Artificial Neural Networks (ANN) have been used for the stock market analysis in multiple ways. Most often they are applied to predict stock prices [2-8] and to analyze data patterns [9-12]. Their wide applications are based mainly on the supervised learning approach, where the input data for the ANN is labelled with the expected output (established by the designer). This way the network can be trained to correctly react on particular data vectors (further called examples). Such an application requires from the investor a careful analysis of historical data and identification of "buy" or "sell" signals in the past. Accuracy of the processing influences effectiveness

${ }^{1}$ The work was partially supported by the Institute of Radioelectronics, Warsaw University of Technology, (statutory grant) 
of the decision making module and accurate categorization of both types of signals. The unsupervised learning ANN is used to find dependencies in the past data without any information about the decision that should be made at the specific time instant. Generation of categories is the subsequent step after grouping similar examples into clusters. Such a methodology was successfully used in classification problems such as diagnostics of analog systems [13], voice [14] or handwritten text recognition [15].

The stock market analysis is difficult as the system itself is complex and mostly of the stochastic nature. There are many factors influencing behavior of shares' prices, some of them are still to be determined.

Construction of the ANN requires providing parameters supporting the learning process. The drawback of the approach is that their optimal values are unknown during the training phase. Therefore the detailed analysis of each problem must be done separately, requiring significant amount of time. Solutions for the stock market require selecting the strategy achieving the highest money returns in the shortest training time. The methodology of the AIbased investment support system is presented in Fig. 2. The decision about what to do with the particular shares in the present moment ("buy", "sell" or "do nothing") is made based on knowledge extracted from the history data and the developed investment strategy. The important part of this process is feature extraction, i.e. calculation of technical analysis factors based on the daily share prices.

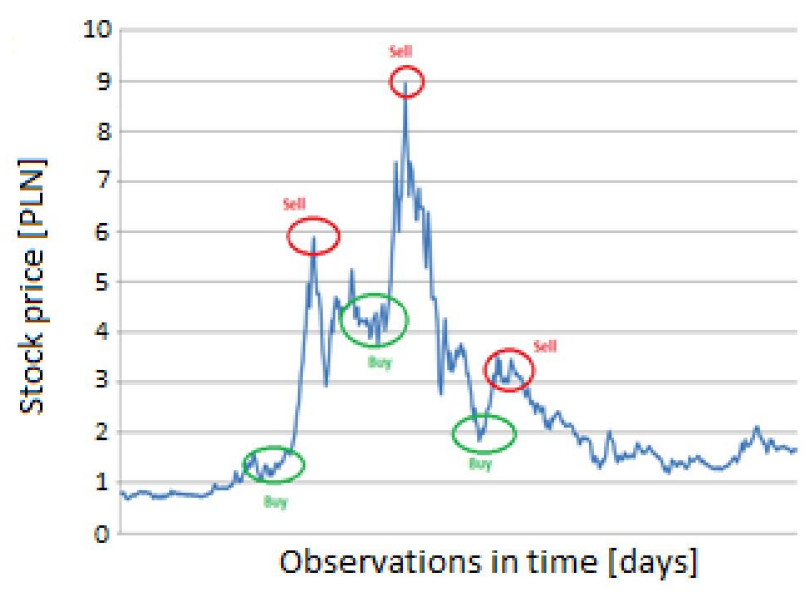

Fig. 1 - Stock prices of the sample company over time from May 2007 to September 2016 (data from hossa.pl)

Every investor aims at maximizing his/her profit. The simplest way to achieve this target is to buy shares cheap and sell them high. The problem is to select the proper moment to do so. In practice, the emergence of significant price drops (abrupt decrease) usually leads to the up adjustment (green points in Fig. 1). This means the share price is evaluated below its value, so investors eagerly buy them. This leads to the subsequent share price increase. Analogously, the emergence of large price ups (abrupt increase) leads to the down adjustment (red points in Fig. 1). These are time instants to be predicted by the investor. The strategy presented in this paper is aimed at maximizing profits using the ANN working with the specific amount of learning data. The purpose of training is to extract knowledge from the past data combined with the investment strategy allows for maximizing the profit in the future (see Fig. 2). To initially assess the quality of the extracted knowledge, the available history data must be divided into the training and testing subsets. They are used for knowledge extraction and testing of its generalization abilities, respectively.

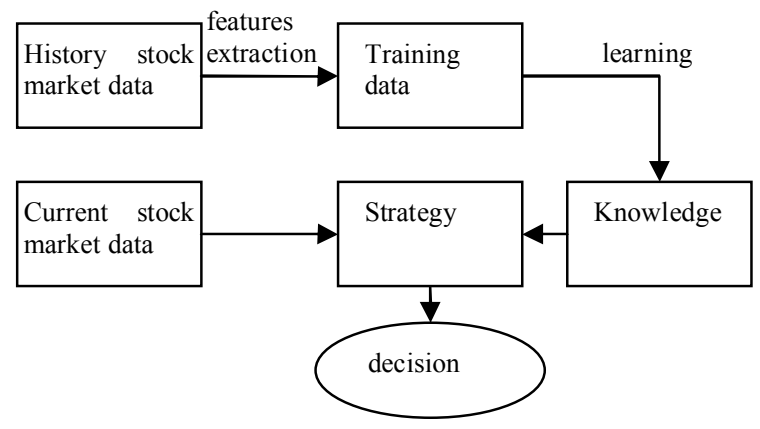

Fig. 2 - Methodology for the investment support using the AI algorithms

As the results of the training, neurons (computational units) with weights adjusted to the problem are able to react on the groups similar features' vectors (defining the condition of the selected company on the particular day). These neurons are subsequently labeled with the category of the desired investment action (as stated above). During the testing, each vector is processed by the SOM and the label of the neuron reacting strongest to its content is presented as the investment suggestion.

\section{METHODOLOGY OF DATA ANALYSIS}

The aim of this study was to determine the relation between the parameters of the SOM and its performance (measured as RoR (1)) for the investment strategy built for the network.

$$
R o R=\frac{v_{t}-v_{t-1}}{v_{t-1}} \cdot 100 \%
$$

where $v_{t}$ is the value of the investment at the end and $v_{t-1}$ is the value of the investment at the starting 
point. The value of RoR is to be maximized during the strategy application.

The unsupervised learning-oriented, one-layered network (Fig. 3) was implemented here, where neurons are trained to react on the presented examples (by changing weights connecting them with corresponding inputs). The weights of neurons reacting to the specific examples in the strongest way are modified to react to them even stronger. The adjustable parameters of the network are:

- size and dimensions of the SOM, i.e. the number of neurons and their positions on the plane (square or rectangular),

- conscience mechanism, allowing for increasing the number of units competing during the training,

- number of neurons trained at the same time (either only the strongest one or the group of units in its vicinity as well),

- learning coefficient, influencing speed of training.

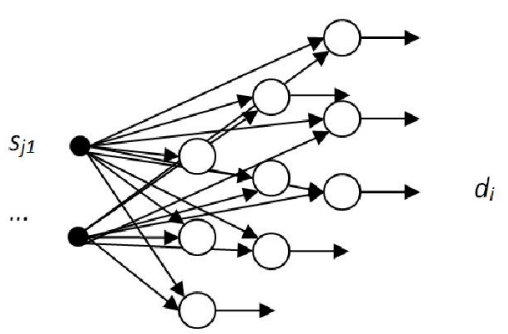

Fig. 3 - Self Organising Map structure

Conducted experiments allowed for verifying all parameters separately. The Matlab 2014 computing environment with Neural Networks toolbox was used for this purpose. The stock market data for companies listed on the Warsaw Stock Exchange were used as the case study. All analyzed companies are or were a part of the WIG20 index (updated on 16.09.2016), which contains the largest twenty companies based on their market capitalization and trading value of shares. These are in particular: Asseco Poland, BRE Bank, Bank BZWBK, Eurocash, Lotos, GTC, Bank Handlowy, KGHM, Bank Pekao, PGNiG, PKN Orlen, Bank PKO BP, Synthos and TPSA. All available data for each company were used for computations. The number of observations in every case is different because dates of entering the stock market were different for each company.

The separate investment strategy was applied to a particular case, as each company is different and the SOM should learn specific patterns provided to its input. Therefore the network is trained only by data for the particular company. During the learning phase, each pattern is processed by all neurons in the SOM. Their response is calculated as the distance between values of the input parameters and weights of the subsequent neurons. The one with the smaller distance is the winner, as its reaction to the vector is the most intense. The next step depends on the selected learning strategy. In the Winner Takes All (WTA) only its weights are modified to react even stronger to this vector. In the Winner Takes Most (WTM), also neurons close to the winner are trained. The purpose of this process is to train particular neurons to react to specific examples ("detect" them). The trained network is further used to classify vectors of features not available during learning.

\section{INVESTMENT STRATEGY DETAILS}

The SOM-based investment strategy is the classification module generating one of three signals on the output as the reaction to the input vector:

- purchase of shares for all available money $(\mathrm{P})$;

- sale of all possessed shares (S);

- hold (do nothing) (H).

The $\mathrm{P}$ and $\mathrm{S}$ signals cause buying or selling all available shares of the selected company, which is the typical approach in the investment strategy development. At the beginning, the investor possesses the initial value of 10,000 Currency Units (CU). The strategy includes the $0.4 \%$ commission on each transaction and excludes the possibility of short selling (i.e. selling shares, although the investor does not possess them - as the strategy is tested in the simulated environment, such operations are possible).

The strategy is generated during the training process. For that purpose, the data set $D$ is required, containing $n$ vectors (examples) $\boldsymbol{x}$, each with $k$ features (in general, real or integer numbers) $f_{i}$.

$$
\boldsymbol{x}=\left[\begin{array}{llll}
f_{1} & f_{2} & \cdots & f_{k}
\end{array}\right]
$$

Features are values calculated based on the daily stock prices (opening, closing, minimum, maximum, etc.) collected for each considered company.

For each example $k=18$ features were used, determining the number of SOM inputs. They include the most popular technical analysis indicators. All processed data were downloaded from financial web portals (such as bossa.pl). The analyzed features are as follows (details of their interpretation can be found in [16-17]):

1-4. the last four 1-day logarithmic Rate of Returns, i.e. RoR calculated as the logarithm of the ratio between the share price $v_{t}$ from today and the share price $v_{t-1}$ from the previous day (3);

$$
R o R_{t-1}=\ln \left(\frac{v_{t}}{v_{t-1}}\right)
$$


5-6. the last two 2-day logarithmic RoR, calculated analogously to (3);

7. the actual gradient of 5-day price trend (4);

$$
m=\frac{\sum_{t=1}^{n}\left(x_{t}-\bar{x}\right)\left(v_{t}-v\right)}{\sum_{t=1}^{n}\left(x_{t}-\bar{x}\right)^{2}}
$$

where $x_{t}$ are consecutive points in time, while $y_{t}$ - stock prices, and $n$ is the analyzed number of days (here 5).

8. the gradient of 5-day price trend from the previous 4 days (4);

9. the actual gradient of 10-day price trend (4);

10. dynamics of change for 1-day logarithmic RoR (5);

$$
\Delta R o R=R o R_{t-1}-R_{o} R_{t-2}
$$

11. dynamic of change for 2-day logarithmic RoR (5);

12. dynamics of change for gradient of 5-day price trend;

13. difference between the actual gradient of 5day price trend and the 10-day price trend;

14. Relative Strength Index (RSI) - indicator used in technical analysis determining the strength of the trend;

15. buy and sell signals obtained from MACD (Moving Average Convergence/Divergence) indicator. It examines the convergence and divergence of moving averages, and is the difference between long-term and short-term values of the exponential average;

16. difference between the last closing price and Moving Average;

17. 14-day Rate of Change $(\mathrm{RoC})$, i.e. the percentage change of the price of the current session to the price from 14 sessions before;

18. Commodity Channel Index (CCI), measuring the deviation of the price of a share from its mean value recognized statistically.

Training consists in presenting subsequent vectors to the SOM, for which the responses are calculated. The $j$-th neuron with the weights $w_{j}$ closest to the training example is considered the winner, as its reaction is the strongest to this vector. The $w_{j}$ vector is modified to make the unit susceptible to this example even stronger.

$$
\boldsymbol{w}_{j}=\boldsymbol{w}_{j}+\eta \cdot p
$$

where $\eta$ is the learning coefficient and $p$ is the direction of correction (increase or decrease).

After presenting all examples from the set $D$, the process is repeated from the beginning. This way the particular neurons are specialized to react exclusively to the particular examples. After training, the SOM contains four groups of neurons. Three of them represent examples for which one of the investment signals are generated $(\mathrm{P}, \mathrm{S}$ or $\mathrm{H})$. The fourth group contains neurons not generating any signal (not responding to any example). As this effect is considered negative, such a group should be as small as possible. Details of the training algorithm are in [18-19].

For each training process, the maximum number of iterations (i.e. presentations of the whole set to the network - training epochs) was the same and equal to 2000. This was the only stopping condition applied. Because the initial weights are selected randomly, every network was constructed and trained 10 times in the constant conditions to verify repeatability of results.

After training, the network is evaluated using additional examples not present in the set $D$. Accuracy of the investment strategy is measured by the RoR calculated on these examples.

\section{SOM PARAMETERS}

The following section presents details of the subsequent SOM parameters, which had to be verified during the research. To make the evaluation as accurate as possible, in each experiment only one parameter was changed. This way the influence of the subsequent SOM configurations on the network accuracy was determined.

\subsection{SOM DIMENSIONS}

The optimal number of neurons in SOM determines the theoretical maximum number of categories to generate during training. This value is related to the size of learning data. The aim is to find the best average number of observations from the learning set per single neuron. This significantly affects the classification accuracy, increasing the resolution and the ability to distinguish between various examples. If the number of neurons is too small and the processed group of vectors is too diverse, dissimilar examples will be assigned to the same cluster. For too many neurons, the number of clusters may be excessive, containing small number of examples. In both cases "inactive" neurons may exist in the network, not responding to any particular examples, which is also the negative effect.

For each company selected for the study, seven investment strategies based on SOM with different sizes were simulated. Each size depends on the number of training examples available for the company and variability in data. For instance, the set of examples scattered and forming clusters distant from each other requires the greater number of 
neurons than the set of the same cardinality, but with examples of small variability. Networks were trained using the WTM strategy (see Section 5.3), where the group of neurons in the vicinity of the winning unit is also trained. This mechanism requires defining the link distance, i.e. the neighborhood. In each network the neurons were arranged in the shape as close to the square as possible. Results in Table 1 show the optimal number of units for each company. The column headers indicate the tested values of average vectors per neuron, which will require different number of neurons for the particular company (as each contains the different number of samples). The rows contain the subsequent number of neurons in each network for the subsequent companies (ensuring the particular average value of vectors per neuron). Comparison between the applied networks is discussed in Section 6.

Table 1. SOM sizes used for each company

\begin{tabular}{|c|c|c|c|c|c|c|c|}
\hline \multicolumn{8}{|c|}{ A varaoe number of yectors ner neuron } \\
\hline Company & 150 & 75 & 50 & 40 & 30 & 20 & 15 \\
\hline asseco & 15 & 30 & 45 & 55 & 75 & 110 & 150 \\
\hline bre & 20 & 45 & 70 & 85 & 115 & 170 & 230 \\
\hline bzwbk & 20 & 45 & 65 & 85 & 110 & 170 & 225 \\
\hline eurocash & 4 & 8 & 11 & 15 & 19 & 28 & 38 \\
\hline lotos & 3 & 5 & 7 & 9 & 12 & 18 & 24 \\
\hline gtc & 5 & 10 & 15 & 20 & 25 & 40 & 50 \\
\hline handlowy & 15 & 30 & 50 & 60 & 80 & 125 & 165 \\
\hline $\mathrm{kghm}$ & 15 & 30 & 50 & 60 & 80 & 125 & 165 \\
\hline pekao & 15 & 30 & 45 & 55 & 75 & 110 & 150 \\
\hline pgnig & 3 & 5 & 8 & 10 & 15 & 20 & 27 \\
\hline pkn orlen & 12 & 25 & 40 & 50 & 60 & 95 & 125 \\
\hline pko bp & 4 & 8 & 13 & 16 & 21 & 31 & 40 \\
\hline synthos & 4 & 8 & 12 & 15 & 20 & 30 & 40 \\
\hline tpsa & 15 & 30 & 40 & 50 & 70 & 105 & 140 \\
\hline
\end{tabular}

\subsection{CONSCIENCE MECHANISM}

Introduction of the conscience mechanism was expected to increase the accuracy of the SOM classification. As in Section 5.1, it is expressed by the percentage RoR from the investment strategy. As a comparison, the results obtained in Section 5.1 were used.

The conscience increases the number of neurons taking part in the learning process. After winning, the particular neuron remains inactive (rests) during a few next presentations of examples. This gives the chance for other units to respond to the next learning patterns and modify their weights. The function introduces the potential $p_{i}$ for the $i$-th neuron, modified after presenting each input $\boldsymbol{x}(7)$, according to the relationship:

$$
p_{i}(k+1)=\left\{\begin{array}{ccc}
p_{i}(k)+\frac{1}{n} & \text { for } & i \neq w \\
p_{i}(k)-p_{\min } & \text { for } & i=w
\end{array}\right.
$$

where $k$ is the number of the iteration in the training algorithm and $w$ is the index of the winning neuron.

The value of $p_{\min }$ is the minimum potential supporting the $i$-th neuron to compete with other neurons during learning process. Each unit with the value of potential below $p_{\text {min }}$, is excluded from calculations. This prevents the particular neuron from dominating the network and adjusting its weights to most of examples. The maximum value of the potential is limited to 1 . Selection of the $p_{\text {min }}$ value affects the neuron's ability to learn. When $p_{\min }=0$, the winning neuron may respond to another learning pattern. If $p_{\min }=1$, the neurons win consecutively, because at any moment there is only one ready to learn. In practice, good results were obtained for $p_{\min }=0.75-$ see $[19,20]$.

Results obtained by the conscience-based investment strategies are expected to give better RoR that the ones without it. The mechanism allows less active neurons to modify their weights, which causes that each neuron to respond to more similar number of vectors. This may shift the best average number of samples for neuron in case of these networks. This is because there is the smaller chance for the appearance of "inactive" units in networks with larger number of neurons.

\subsection{LEARNING STRATEGY}

The effect of using the WTA (Winner Takes All) learning method instead of WTM (Winner Takes Most) was also examined. The former causes that only one, winning neuron has adjusted weights. In the WTM approach, additionally to the winning neuron its neighbors are trained as well. Units from the neighborhood located closer to winning one adjust weights more intensely than neurons located farther. The neighborhood from Section 5.1 was defined as the link distance function and set to 3 . Using WTM causes that more neurons adjust their weights and decreases the chance that some of them do not generate any investment signals.

\subsection{CORRELATION IN DATA}

Features presented in Section 4 were selected because of their wide usage in the technical analysis, but for the strategy described in the paper many of them could be redundant. Some features may present similar information, so their existence in the set $D$ is unnecessary. To check such dependences, correlation between pairs of features was checked. On this basis two reduced data sets were obtained, each containing less features than the original set 
used in Section 5.1. The first one $\left(D_{17}\right)$ contains 17 features, without $f_{13}$, having the correlation indicator with $f_{12}$ higher than 0.9 in at least $80 \%$ of companies. The second subset $\left(D_{11}\right)$ contains eleven features, without $f_{5}, f_{6}, f_{9}, f_{11}, f_{13}, f_{14}$ and $f_{16}$ (see Section 4). They have the correlation indicator higher than 0.7 for at least $60 \%$ of companies. Comparing strategies built on these two datasets with strategies from Section 5.1 shows the significant impact of correlated features in data set, described in Section 6.4 .

\section{EXPERIMENTAL RESULTS}

\subsection{SOM DIMENSIONS}

To check the impact of each SOM parameter on its performance, training and testing processes were repated and concluded with the RoR calculation. These values were then compared to reveal the optimal combination of parameters, ensuring the most effective investment strategy Firstly, networks of different sizes, based on the average number of learning patterns per neuron, were tested. Next, for each company the SOM size was verified, according to three measures: minimal, maximal and average RoR. Table 2 contains percentages of strategies for companies based on particular SOMs' configurations (Table 1) with the best results in each category. Quality factors include minimum, maximum and average RoR, calculated for ten repeats of the experiment (i.e. training the network from scratch and testing it on the available data). Because the SOM training starts with randomly selected weights, experimental results must be interpreted statistically. The selected measures show, how many times the particular SOM gives the optimal RoR for all companies. Values in the particular column sum up to 100 percent.

Table 2. Influence of the network dimensions on the investment strategy performance

\begin{tabular}{|l|c|c|c|}
\hline \multirow{2}{*}{$\begin{array}{c}\text { Avg. } \\
\text { vectors } \\
\text { per } \\
\text { neuron }\end{array}$} & \multicolumn{2}{|c|}{$\%$ of best strategies for companies } \\
\cline { 2 - 4 } & Min. RoR & Max.RoR & Avg. RoR \\
\hline 150 & $52.97 \%$ & $24.58 \%$ & $44.95 \%$ \\
\hline 75 & $10.38 \%$ & $2.85 \%$ & $10.06 \%$ \\
\hline 50 & $6.52 \%$ & $18.33 \%$ & $14.52 \%$ \\
\hline 40 & $12.92 \%$ & $12.93 \%$ & $12.76 \%$ \\
\hline 30 & $1.91 \%$ & $18.19 \%$ & $1.69 \%$ \\
\hline 20 & $0.92 \%$ & $15.59 \%$ & $1.31 \%$ \\
\hline 15 & $14.38 \%$ & $7.53 \%$ & $14.71 \%$ \\
\hline
\end{tabular}

Results of this experiment show that networks with large number of learning patterns per neuron perform better in the investment strategies. The average RoR for networks with 150 learning vectors per neuron is better than the rest of structures for nearly $45 \%$ of companies.

Data available for the subsequent shares were of different sizes and the relative network size was different for all of them. Larger SOMs with the larger number of patterns per neuron behaved more stable than the smaller ones. Such networks in individual cases allowed for obtaining better RoR, but their variations were much higher. The chance to get better or worse RoR from the investment strategy was similar. Usability of these strategies is therefore limited.

\subsection{CONSCIENCE MECHANISM}

Influence of the conscience mechanism on the network efficiency is presented in Table 3. The percentage of companies, for which SOMs with the conscience mechanism perform better, is 59\% (based on the average RoR). In 12\% cases SOMs obtain comparable results, while in $28 \%$ of cases SOMs without the conscience mechanism perform better. Similar results were obtained based on the minimal and maximal RoR. As in Table II, values in subsequent columns are summed up to 100 percent.

Table 3. Influence of the conscience mechanism on the investment strategy performance

\begin{tabular}{|c|c|c|c|}
\hline & \multicolumn{3}{|c|}{$\begin{array}{c}\% \text { of best strategies for } \\
\text { companies }\end{array}$} \\
\hline & $\begin{array}{l}\text { Min. } \\
\text { RoR }\end{array}$ & $\begin{array}{l}\text { Max. } \\
\text { RoR }\end{array}$ & $\begin{array}{l}\text { Avg. } \\
\text { RoR }\end{array}$ \\
\hline $\begin{array}{l}\text { SOM without conscience } \\
\text { performing better }\end{array}$ & $32.27 \%$ & $28.06 \%$ & $28.45 \%$ \\
\hline $\begin{array}{l}\text { Equal outcomes for both } \\
\text { SOM types }\end{array}$ & $13.57 \%$ & $20.3 \%$ & $12.18 \%$ \\
\hline $\begin{array}{l}\text { SOM with conscience } \\
\text { performing better }\end{array}$ & $54.16 \%$ & $51.64 \%$ & $59.37 \%$ \\
\hline
\end{tabular}

Strategies based on networks with the conscience mechanism in general perform better than for networks without it. RoRs from such strategies are higher by average $9 \%$. After including only the cases when these strategies were better, this average values rises up by 17 percent. The average RoR for strategies without the implemented conscience mechanism was better only by 5 percent. This proves the usability of the proposed networks, but requires the careful selection of their parameters and repeated training/testing scheme.

\subsection{LEARNING METHOD}

Influence of the learning method on the strategy performance is presented in Table 4. Generally, WTA gives superior results (in almost $50 \%$ cases 
performs better based on the average RoR), but in almost $43 \%$ of cases based on minimal RoR, the WTM method performs better (at almost $41 \%$ for WTA). Also, small differences based on the maximal RoR suggest that learning method doesn't have impact on the investment strategy quality or the tested pool of companies is too small. Expanding it should be considered in the future research. Also, different neighborhood should be evaluated.

Table 4. Influence of the learning methods on the investment strategy performance

\begin{tabular}{|c|l|l|l|}
\cline { 2 - 4 } \multicolumn{1}{c|}{} & \multicolumn{3}{c|}{$\%$ of best strategies for companies } \\
\cline { 2 - 4 } \multicolumn{1}{c|}{} & Min. RoR & Max. RoR & Avg. RoR \\
\hline $\begin{array}{c}\text { WTM performing } \\
\text { better }\end{array}$ & $42.86 \%$ & $39.80 \%$ & $35.71 \%$ \\
\hline $\begin{array}{c}\text { Equal outcomes for } \\
\text { both methods }\end{array}$ & $16.33 \%$ & $18.37 \%$ & $15.31 \%$ \\
\hline $\begin{array}{c}\text { WTA performing } \\
\text { better }\end{array}$ & $40.82 \%$ & $41.84 \%$ & $48.98 \%$ \\
\hline
\end{tabular}

\subsection{CORRELATION IN DATA}

Performance comparison of methods built on data sets with different number of features $|f|$ is presented in Table 5. Clearly, strategies built on data without correlated features perform better than others $(62,67 \%$ based on the average RoR). Returns from such strategies are higher by average $7 \%$. After including only the cases when these strategies were better, this average values rises up to $13 \%$. Analysis of correlation between features can easily improve quality of the investment strategy.

Table 5. Results of Experiment - Corrrelation in data

\begin{tabular}{|c|c|c|c|}
\cline { 2 - 4 } \multicolumn{1}{c|}{} & \multicolumn{3}{c|}{$\%$ of best strategies for companies } \\
\cline { 2 - 4 } \multicolumn{1}{c|}{} & Min. RoR & Max. RoR & Avg. RoR \\
\hline $\begin{array}{c}D_{18} \text { with highest } \\
\text { RoR }\end{array}$ & $14.71 \%$ & $28.33 \%$ & $24.00 \%$ \\
\hline $\begin{array}{c}D_{17} \text { with highest } \\
\text { RoR }\end{array}$ & $16.18 \%$ & $15.00 \%$ & $13.33 \%$ \\
\hline $\begin{array}{c}D_{11} \text { with highest } \\
\text { RoR }\end{array}$ & $69.12 \%$ & $56.67 \%$ & $62.67 \%$ \\
\hline
\end{tabular}

Exclusion of correlated data from the data set also speeds up learning process due to the smaller number of features, which is the additional reason to perform data analysis and carefully choose features to the particular strategies.

Comparison between the SOM and other approaches used to make investment strategies is difficult, as they (especially supervised learning ANNs, such as multi-layered perceptrons) process learning data differently. The future work should include the direct comparison between such architectures, using measures presented here.

\section{CONCLUSIONS}

In this paper the influence of the SOM parameters on the accuracy of investment strategies in the stock market were verified. The best single SOM-based investment strategy is the one with WTA learning method and the conscience mechanism implemented. Its size corresponds to the learning data per the single neuron. Here the optimal value is, in average, 150 vectors. From data set the correlated features should be removed to speed up performance of neural network and increase the RoR. Future works require comparison between alternative AI-based approaches and the implementation of the ensemble of classifiers, for instance combining multiple configurations of SOMs, all trained on the same data.

\section{REFERENCES}

[1] P. Kossakowski, P. Bilski, "Application of selforganizing maps to the stock exchange data analysis," in Proceedings of the $8^{\text {th }}$ IEEE International Conference on Intelligent Data Acquisition and Advanced Computing Systems: Technology and Applications (IDAACS'2015), Warsaw, Poland, September 24-26, 2015, pp. 208-2013.

[2] E. Gurusen, G. Kayakulu and T. U. Daim, "Using artificial neural network model in stock market index prediction," Expert Systems with Applications, Vol. 38, Issue 8, pp. 1038910397, 2011.

[3] V. Turchenko, P. Beraldi, F. De Simone, L. Grandinetti, "Short-term stock price prediction using MLP in moving simulation mode," in Proceedings of the 6th IEEE International Conference on Intelligent Data Acquisition and Advanced Computing Systems (IDAACS'2011), Prague, Czech republic, 15-17 September 2011, pp. 666-671.

[4] X. Lin, Z. Yang and Y. Song, "Short-term stock price prediction based on echo state networks," Expert Systems with Applications, Vol. 36, pp. 7313-7317, 2009.

[5] X. Zhu, H. Wang, L. Xu and H. Li, "Predicting stock index increments by neural networks: The role of trading volume under different horizons," Expert Systems with Applications, Vol. 34, pp. 3043-3054, 2008.

[6] Y. Kara, M. A. Boyacioglu and O. K. Baykan, "Predicting direction of stock price index movement using artificial neural networks and support vector machines: the sample of the Instabul Stock Exchange," Expert Systems with Applications, Vol. 38, pp. 5311-5319, 2011.

[7] M. Qiu, Y. Song, "Predicting the direction of stock market index movement using an 
optimized artificial neural network model," PLoS ONE, Vol. 11, Issue 5, e0155133, 2016. doi:10.1371/journal.pone.0155133.

[8] Z. Guo, H. Wang, J. Yang, D.J. Miller, "A stock market forecasting model combining twodirectional two-dimensional principal component analysis and radial basis function neural network," PLoS ONE, Vol. 10, Issue 4, e0122385, 2015. doi:10.1371/journal.pone. 0122385 .

[9] P. Bilski, "Analysis of the stock exchange waveforms similarity using the clustering method," Polish Journal of Environmental Studies, Vol. 18, No. 5B, pp. 13-20, 2009.

[10] J. Vesanto, E. Alhoniemi, "Clustering of the self-organizing map," IEEE Transactions on Neural Network, Vol. 11, pp. 586-600, 2000.

[11] E. W. Saad, D. V. Prokhoro, "Comparative study of stock trend prediction using time delay," IEEE Transactions on Neural Networks, Vol. 9, pp. 1456-1470, 1998.

[12] S. Subramanian, U. S. Rao, "Enhancing stock selection in Indian Stock Market using value investment criteria: an application of artificial neural networks," IUP Journal of Accounting Research \& Audit Practices, Vol. 9, pp. 54-67, 2010.

[13] P. Bilski, "Ambiguity groups detection in analog systems diagnostics using SelfOrganizing Maps," in Proceedings of the IMEKO TC10 Workshop, Milan, Italy, June 27-28 2016, pp. 294-299.

[14] C. Estrebou, L. Lanzarini, W. Hasperué, "Voice recognition based on probabilistic SOM," in Proceedings of the Conference: XXXVI Conferencia Latinoamericana en Informática, At Asunción, Paraguay, 2010.

[15] M. G. Kibria and Al-Imtiaz, "Bengali optical character recognition using self organizing map," in Proceedings of the International Conference on Informatics, Electronics \& Vision (ICIEV), 18-19 May 2012, 10.1109/ICIEV.2012.6317479.
[16] J. J. Murphy, Technical Analysis of the Financial Markets, Prentice Hall Press, 1999.

[17] H. Kwaśnicka, M. Ciosmak, "Itelligent techniques in stock analysis," Advances in Intelligent and Soft Computing, Vol. 10, pp. 195-208, 2001.

[18] R. Tadeusiewicz, Neural Networks, Akademicka Oficyna Wydawnicza, 1993. (in Polish).

[19] A. P. Engelbrecht, Computational Inteligence: An Introduction, John Wiley \& Sons, 2007.

[20] S. Osowski, Neural Networks in Algorithmic Approach, WNT, 1996. (in Polish).

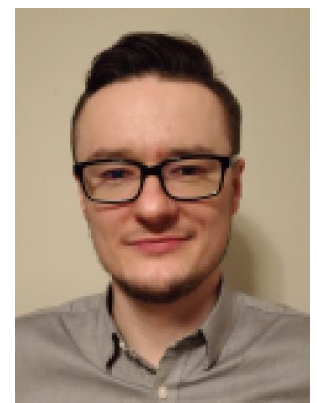

Piotr Kossakowski, MSc was born in 1985 in Ziębice. He graduated from Warsaw University of Life Sciences obtaining MSc degree in 2008. Currently is working as Senior Data Analyst in Risk Department in Bank Millennium SA. His main scientific interests include applications of neural networks in economics, banking and finance

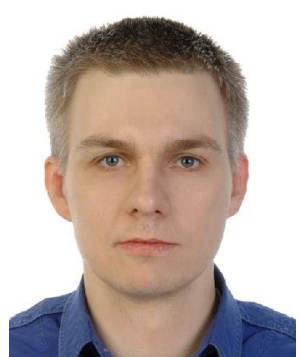

Piotr Bilski, PhD was born in 1977 in Olsztyn, Poland. He graduated from Warsaw University of Technology, Institute of Radioelectronics, obtaining MSc degree in 2001 (with honors), $P h D$ degree in 2006 (with honors) and DSc degree in 2014. Currently he is an Associate Professor in the Institute of Radioelectronics and Multimedia Technology, Warsaw University of Technology. His main scientific interests include diagnostics of analog systems, design and analysis of virtual instrumentation, application of artificial intelligence and machine learning methods in environmental sciences. He is the member of IEEE, IMEKO TC10 and POLSPAR, and reviewer for such journals like Measurement, IEEE Transactions on Instrumentation and Measurement, Expert Systems with Applications. 